\title{
BOUNDARY VALUE PROBLEMS FOR ELASTIC HALF-PLANES POSED IN TERMS OF STRESS AND DISPLACEMENT ORIENTATIONS
}

\author{
A. N. GALYBIN \\ The Schmidt Institute of Physics of the Earth (IPE RAS), Moscow, Russia.
}

\begin{abstract}
This study investigates solvability of boundary value problems of plane elasticity formulated in terms of principal directions of the stress tensor and the orientations of the displacement vector. The analysis of solvability is performed by using the following approach. Firstly, boundary values of the complex potentials are represented by the Cauchy-type integrals with unknown density. Then a system of singular integral equations is obtained by satisfying particular boundary conditions. This system is further reduced to the system of the Riemann boundary value problems for the determination of sectionally holomorphic functions. Solvability of the Riemann problems is investigated by calculating their indexes. This allows one to determine the number of linearly independent solutions and hence the number of arbitrary parameters entering into the general solution.

Two novel formulations have been investigated for the case of elastic half-planes. In both cases the initial system of equations has been reduced to the form that allow for successive solution of its equations. Keywords: boundary value problems, complex potentials, plane elasticity, singular integral equations
\end{abstract}

\section{INTRODUCTION}

In geomechanics the boundary values of stresses and/or displacements are often unknown on the boundary, which makes it impossible to apply classical formulations accepted in solid mechanics. However even incomplete information, such as orientations of principal stresses or displacements can serve as possible boundary conditions, BC. Accordingly, several non-classical formulations of elastic boundary value problems, BVP, employing BC in terms of orientations of principal stresses, displacements or tractions have been investigated in Refs. [1-6]. The mentioned studies are mostly dedicated to the investigation of solvability of the BVP with incomplete BC, which leads to non-unique solutions. The latter is proved by using the following approach. The complex potentials are presented by the Cauchy integrals with unknown densities. Their boundary values are found by employing the Sokhotski-Plemelj formulas. These are used to express the orientations of tractions, principal stresses and displacements on the contour in the form that contains two unknown complex valued functions. Further on, one of these functions is excluded by imposing the condition of continuity of the stress vector across the contour. Then by using two scalar BC in terms of the orientations one can derive a system of two real singular equations, SIE. These are combined into one linear complex SIE of the general form that contains the unknown function, its conjugate, the singular and regular integrals and their conjugates. An approach suggested by Gakhov [7] is used for analysing the solvability of the complex SIE. It assumes separation of the dominant part of the complex SIE followed by its conversion into the equivalent Riemann problem. The solvability of the latter depends on the index of the coefficient of the Riemann problem that is calculated through the increment of its argument (divided by $2 \pi$ ) in traversing the contour anticlockwise. If the index is positive then the Riemann problem has a finite number of solutions that is proportional to the index, otherwise the BVP does not have solutions in the class of holomorphic functions. This approach can also be adopted for numerical solving complete complex SIEs [6]. 
It is shown in Refs. [1-6] that the dominant SIE corresponds to the case of elastic halfplane regardless of the $\mathrm{BC}$ used. Therefore we further consider the dominant SIE only. However, in contrast to the previously used approach we deal with two real SIEs. The reason behind this is to build the successive solutions of the equations in the system for the problems that do not directly allow for the application of the technique mentioned above because of the presence of the derivatives of the unknown functions in the complex dominant SIE.

\section{BOUNDARY VALUES OF STRESSES AND DISPLACEMENTS}

Let us introduce the following notations based on the Kolosov formulas [8]

$$
\begin{gathered}
D \equiv \frac{\sigma_{22}-\sigma_{11}}{2}+i \sigma_{12}=\tau_{\max } \exp (i \alpha), \quad \alpha=\pi-2 \theta, \\
W \equiv 2 G\left(u_{1}+i u_{2}\right)=\omega \exp (i \beta) .
\end{gathered}
$$

Here $D$ is the complex stress deviator function, $\sigma_{11}, \sigma_{12}, \sigma_{22}$ are the components of the stress tensor $\tau_{\max }=\left(\sigma_{1}-\sigma_{2}\right) / 2 \geq 0$ is the maximum shear stress, $\sigma_{1}, \sigma_{2}$ are major and minor principal stresses respectively, the angle $\theta=\frac{1}{2} \operatorname{arctg} \frac{2 \sigma_{12}}{\sigma_{11}-\sigma_{22}}$ specifies the principal direction (the angle between $\sigma_{1}$ and the real axis), thus $D=-\tau_{\max } \exp (-2 i \theta) ; W$ is the complex displacement vector, $G$ is the shear modulus, $u_{1}, u_{2}$ are the displacement components, $\omega \geq 0$ and $\beta$ are respectively the modulus and the argument of the function $W$.

On the real axis, denoted as contour $\Gamma$ further on, the boundary values of the functions $D$ and $W$ are found as follows $[1,4]$

$$
\begin{gathered}
2 D^{ \pm}=\mp\left(g^{\prime}+\bar{g}^{\prime}\right)-\mathbf{S}\left(g^{\prime}+\bar{g}^{\prime}\right), \\
2 W^{ \pm}= \pm\left(\kappa^{ \pm}+1\right) g+\left(\kappa^{ \pm}-1\right) \mathbf{S}(g) .
\end{gathered}
$$

Here the superscripts + /- stay for the boundary values for the upper/lower half-planes respectively, $\kappa^{ \pm}$are the Muskhelishvili's constants (they can be different for different half-planes) and the following notation for the singular integral was introduced

$$
\mathbf{S}(g)=\frac{1}{\pi i} \int_{\Gamma} \frac{g(t)}{t-\zeta} d t=\frac{1}{\pi i} \int_{-\infty}^{\infty} \frac{g(t)}{t-\zeta} d t, \quad \zeta \in \Gamma \Leftrightarrow \operatorname{Im} \zeta=0 .
$$

The complex operator has the following properties

$$
\begin{aligned}
& \mathbf{S}[\mathbf{S}(g)]=g, \\
& \overline{\mathbf{S}(g)}=-\mathbf{S}(\bar{g}) .
\end{aligned}
$$

The former is valid for any closed contour, while the latter is valid only for the real axis.

It should be noted that the representations in (3) and (4) are given through the only complex valued function $g$ that is proportional to the unknown displacement jump across the contour. Meanwhile they automatically satisfy the condition of continuity of the stress vector, $p$, across the real axis, i.e. the condition

$$
p^{+}(\zeta)-p^{-}(\zeta)=0, \quad \operatorname{Im}(\zeta)=0 .
$$

The following BC are used further on 


$$
\begin{array}{ll}
\arg D^{ \pm}=\alpha^{ \pm}(\zeta), & \zeta \in \Gamma, \\
\arg W^{ \pm}=\beta^{ \pm}(\zeta), \quad \zeta \in \Gamma .
\end{array}
$$

Taking into account the properties of the singular operator one can express (9)-(10) in terms of the sought function $g$ by using (3)-(4) as follows

$$
\begin{array}{r}
{\left[\mp\left(g^{\prime}+\bar{g}^{\prime}\right)-\mathbf{S}\left(g^{\prime}+\bar{g}^{\prime}\right)\right] \exp \left(-i \alpha^{ \pm}\right)-\left[\mp\left(g^{\prime}+\bar{g}^{\prime}\right)+\mathbf{S}\left(g^{\prime}+\bar{g}^{\prime}\right)\right] \exp \left(i \alpha^{ \pm}\right)=0,} \\
{\left[ \pm\left(\kappa^{ \pm}+1\right) g+\left(\kappa^{ \pm}-1\right) \mathbf{S}(g)\right] \exp \left(-i \beta^{ \pm}\right)-\left[ \pm\left(\kappa^{ \pm}+1\right) \bar{g}-\left(\kappa^{ \pm}-1\right) \mathbf{S}(\bar{g})\right] \exp \left(i \beta^{ \pm}\right)=0 .}
\end{array}
$$

Note that equations (11)-(12) are real valued.

It is convenient to introduce the real and imaginary parts of the sought function $g$ by using the notations

$$
g=\mathrm{M}+i \mathrm{~N}, \quad g^{\prime}=\mu+i v, \quad \mu=\mathrm{M}^{\prime}, \quad v=\mathrm{N}^{\prime} .
$$

Then (11) can be presented in the form

$$
\pm i \mu \sin \left(i \alpha^{ \pm}\right)-\cos \left(i \alpha^{ \pm}\right) \mathbf{S}(\mu)=0
$$

We further consider the following BVPs:

1. Problem $(\alpha, p)$ assumes that boundary values of the stress orientations in (9) are known and condition (8) is satisfied.

2. Combined problem $(\alpha, p)$ and $(\beta, p)$ assumes that $\mathrm{BC}(9)-(10)$ are satisfied simultaneously on upper/lower boundary and complemented by condition (8).

3. Problem $(\alpha, \beta)$ also assumes BC (8)-(10) but only for upper (or lower) half-plane alone.

It is worth to notice that these problems can be reduced to a single complex SIE. In this case problem $(\alpha, p)$ contains the only real valued function (next section) while other two problems would include both the complex unknown function and its derivative, which makes it impossible the direct application of the technique proposed by Gakhov [7].

\section{PROBLEM $(\alpha, p)$ FOR JOINED HALF-PLANES}

Let us consider a BVP with the $\mathrm{BC}$ in terms of the principal directions of the stress tensor that are specified on the $x$-axis that presents the interface between the upper $\Omega^{+}$and lower $\Omega^{-}$half-planes.

It is assumed that the stress vector is continuous across the interface and the boundary values of the principal directions are different on the $\Omega^{+}$and $\Omega^{-}$boundaries. They can be expressed as the boundary values of the argument of the complex valued function of the stress deviator $\arg D^{ \pm}=\alpha^{ \pm}$. The loads acting on the interface are unknown as well as displacements, body forces are absent, the stresses at infinity are uniform but unknown, or vanish.

In this case the complex dominant SIE can be expressed as follows

$$
\left(2-e^{4 i \theta^{+}}-e^{4 i \theta^{-}}\right) \mu+\left(e^{4 i \theta^{-}}-e^{4 i \theta^{+}}\right) \mathbf{S}(\mu)=0 .
$$

The conditions $\tau_{\max } \geq 0$ should be satisfied, which results in the following inequality 


$$
\sin \left(2 \theta^{+}\right) \sin \left(2 \theta^{-}\right) \geq 0 .
$$

The corresponding Riemann BVP is radically simplified to the following form

$$
e^{-2 i \theta^{+}} \sin \left(2 \theta^{+}\right) A^{+}=e^{-2 i \theta^{-}} \sin \left(2 \theta^{-}\right) A^{-} .
$$

Since the origin and the infinity belong to the $x$-axis, one should seek the solution of the Riemann BVP with the use of the linear fractional function, which leads to the following form of the BVP [7]

$$
A^{+}=\left[e^{-2 i\left(\theta^{+}-\theta^{-}\right)}\left(\frac{x-i}{x+i}\right)^{-\mathrm{K}} G_{0}\right]\left(\frac{x-i}{x+i}\right)^{\mathrm{K}} A^{-}, \quad G_{0}=\frac{\sin \left(2 \theta^{-}\right)}{\sin \left(2 \theta^{+}\right)},
$$

where $\mathrm{K}$, is a non-negative index calculated in accordance with the following formula

$$
\mathrm{K}=\operatorname{Index}\left(e^{-2 i\left(\theta^{+}-\theta^{-}\right)}\right)=\left.\frac{1}{\pi}\left[\theta^{+}-\theta^{-}\right]\right|_{\Gamma} .
$$

As soon as the index of the linear fractional function is equal to unity and the index of $G_{0}$ is zero, then the term is square brackets in the right hand side of (18) has zero index. Therefore the solution of the Riemann BVP assumes the following form, [7]

$$
A^{ \pm}(z)=\frac{P_{\mathrm{K}}(z)}{(z \pm i)^{K}} e^{Y^{ \pm}(z)}, Y^{ \pm}(z)=\frac{1}{2 \pi i} \int_{-\infty}^{\infty} \ln \left[\left(\frac{t-i}{t+i}\right)^{-\mathrm{K}} G(t)\right] \frac{1}{t-z} d t, \operatorname{Im}(z) \neq 0 .
$$

On the $x$-axis one can present the solution as follows

$$
A^{ \pm}=\frac{P_{\mathrm{K}}(z)}{\left(x^{2}+1\right)^{\frac{K}{2}}}\left(\frac{\sin 2 \theta^{-}}{\sin 2 \theta^{+}}\right)^{ \pm \frac{1}{2}} e^{\mp i\left(\theta^{+}-\theta^{-}\right)} e^{\frac{1}{2} \mathbf{S}\left[-\mathrm{K} \ln \left(\frac{t-i}{t+i}\right)+\ln (G(t))\right]}
$$

Let us consider the complex conjugated singular operator in the solution above.

$$
\begin{aligned}
\mathbf{S}\left[-\mathrm{K} \ln \left(\frac{t+i}{t-i}\right)+\ln (G(t))\right] & = \\
& =-\mathbf{S}\left[-\mathrm{K} \ln \left(\frac{t+i}{t-i}\right)+2 i\left(\theta^{+}-\theta^{-}\right)+\ln \left(G_{0}(t)\right)\right]= \\
& =\mathbf{S}\left[-\mathrm{K} \ln \left(\frac{t+i}{t-i}\right)+\ln (G(t))\right]-2 \mathbf{S}\left[\ln \left(G_{0}(t)\right)\right] .
\end{aligned}
$$

Here the latter term in the right hand sine is pure imagine, let us denoted it as

$$
2 \mathbf{S}\left[\ln \left(G_{0}(t)\right)\right]=2 i \chi, \quad \operatorname{Im} \chi=0 .
$$

Then solution of (15) takes the form

$$
\mu=-2 i \sin \left(\theta^{+}-\theta^{-}-\chi\right) \frac{P_{\mathrm{K}}(z)}{\left(x^{2}+1\right)^{\frac{K}{2}}}\left(\frac{\sin 2 \theta^{-}}{\sin 2 \theta^{+}}\right)^{ \pm \frac{1}{2}} e^{\mathbf{S}\left[-\frac{\mathrm{K}}{2} \ln \left(\frac{t-i}{t+i}\right)-i\left(\theta^{+}-\theta^{-}\right)\right]}
$$


It follows from (23) that the exponential term in (24) is real-valued. The coefficients of the polynomial should be real to provide real-valuedness of the solution. Thus, the solution depends on $(\mathrm{K}+1)$ linearly independent real constants.

The solution above determines the boundary values of the complex deviator on the $x$-axis. However this is not sufficient for all points lying outside the $x$-axis. This is evident from the fact that the expression for the complex deviator function can be presented the form [1-3]

$$
D(z, \bar{z})=\frac{-1}{\pi i} \int_{-\infty}^{\infty} \frac{\mu(t)}{t-z} d t+(\bar{z}-z) \frac{1}{2 \pi i} \int_{-\infty}^{\infty} \frac{\mu^{\prime}(t)+i v^{\prime}(t)}{t-z} d t .
$$

Therefore we have just found a partial solution that corresponds to the real part of the sought function $g^{\prime}(x)$. It can be presented as follows

$$
D_{\mu}(z, \bar{z})=-2 \Phi_{\mu}(z)+(\bar{z}-z) \Phi_{\mu}^{\prime}(z)
$$

where the following notation for the holomorphic function has been introduced in terms of the Cauchy integral

$$
\Phi_{\mu}(z)=\frac{1}{2 \pi i} \int_{-\infty}^{\infty} \frac{\mu(t)}{t-z} d t
$$

This solution should be complemented by adding the terms of the form $(\bar{z}-z) \mathrm{X}^{ \pm}(z)$, where $\mathrm{X}^{ \pm}(z)$ are arbitrary holomorphic functions decaying at infinity faster then $z^{-1}$. These functions do not affect the boundary conditions on the $x$-axis and at infinity, and, moreover, the function $D$ remains bi-holomorphic. Thus, the general solution is determined to within an arbitrary holomorphic function, indicating that the problem is substantially underdetermined.

Nevertheless, the analysis above makes sense for applications, since it allows one to determine such important characteristics of the stress state on the interface between the half-planes as the shear stresses and maximum shear stresses. The knowledge of these is important when assessing the onset of the propagation of shear fracture. On the other hand this solution can be combined with boundary conditions of other types, for instance, with the conditions imposed on the curvature of the stress trajectories. For example let us assume homogeneous conditions on the $x$-axis $\alpha^{ \pm}=-2 \theta_{0}^{ \pm}$(conditions $\tau_{\max } \geq 0$ assume to be fulfilled). In this case the Riemann BVP has zero index, hence the functions $A^{ \pm}(z)$ are constants as well as the sought function. Meanwhile this constant is real. Thus, the solution for the boundary values takes the form

$$
D^{ \pm}=C e^{i \alpha^{ \pm}}, \quad \operatorname{Im}(C)=0
$$

This solution coincides with the one obtained in Ref. [1] by using a completely different approach.

Another possibility requires attracting the boundary condition in the orientations of the displacement vector.

\section{COMBINED PROBLEM $(\alpha, p)$ AND $(\beta, p)$ FOR HALF-PLANES}

For simplicity, we consider the case of half-planes made of the same material and assume a pair of boundary conditions (9)-(10). The SIE of the problem takes the form (we omit the indices at the elastic constant and apply the properties of the singular operator) 


$$
e^{-i \beta^{ \pm}}[ \pm(\kappa+1) g+(\kappa-1) \mathbf{S}(g)]-e^{i \beta^{ \pm}}[ \pm(\kappa+1) \bar{g}-(\kappa-1) \mathbf{S}(\bar{g})]=0 .
$$

These two scalar equations can be combined into one complex equation by eliminating $\mathbf{S}(\bar{g})$ as follows

$$
(\kappa+1)\left(e^{-2 i \beta^{+}}+e^{-2 i \beta^{-}}\right) g-2(\kappa+1) \bar{g}+(\kappa-1)\left(e^{-2 i \beta^{+}}-e^{-2 i \beta^{-}}\right) \mathbf{S}(g)=0 .
$$

We suppose that the real part of the unknown function is known from the solution of the previous problem. The complex conjugate function is further represented in terms of the integral of the function $\mu(t)$ as follows

$$
\bar{g}=2 \mathrm{M}-g, \quad \mathrm{M}^{\prime}=\mu .
$$

Then the SIE assumes its final form

$$
\left(2+e^{-2 i \beta^{+}}+e^{-2 i \beta^{-}}\right) g+\frac{\kappa-1}{\kappa+1}\left(e^{-2 i \beta^{+}}-e^{-2 i \beta^{-}}\right) \mathbf{S}(g)=4 \mathrm{M}
$$

This equation is slightly different from SIE (15) and in contrast to (15) is non-homogeneous. Its right hand side is found by integration of (24), which generates one additional arbitrary constant. The latter should be real as the function $\mathrm{M}$ is real. Therefore the right hand side depends on $(\mathrm{K}+2)$ arbitrary real constants.

The coefficient of the associated Riemann BVP is found as follows

$$
G=\frac{\kappa e^{-i \beta^{-}} \cos \beta^{-}+e^{-i \beta^{+}} \cos \beta^{+}}{\kappa e^{-i \beta^{+}} \cos \beta^{+}+e^{-i \beta^{-}} \cos \beta^{-}} .
$$

It is evident that $\operatorname{Index}(G)=0$ because $\kappa \cos ^{2} \beta^{ \pm}+\cos ^{2} \beta^{\mp}>0$, i.e. the real parts of both the numerator and the denominator of the fraction are positive. Therefore the non-homogeneous SIE in (32) has a unique solution. The general solution is found by summing the function $M$ with the real part of the function $g$. Moreover, the number of arbitrary constants remains. As soon as the derivative of $\mathrm{M}$ is required for the stress determination, the general solution depends on up to $(\mathrm{K}+1)$ arbitrary constant entering into the solution for the function $\mu(t)$.

\section{PROBLEM $(\alpha, \beta)$ FOR HALF-PLANE}

The same method as above can be used to find the solution of the BVP formulated in terms of principal stress and displacement orientations. Let us consider the upper half-plane. Then the indices can be omitted and one can write the $\mathrm{BC}$ on the $x$-axis in the form

$$
\begin{aligned}
& \arg D=\alpha, \\
& \arg W=\beta .
\end{aligned}
$$

We still seek solutions that provide continuity of the stress vector across the real axis, but now we use the scalar equations for the upper half-plane. Then the system of SIE assumes the form

$$
\begin{aligned}
& e^{-i \alpha}[\mu+\mathbf{S}(\mu)]-e^{i \alpha}[\mu-\mathbf{S}(\mu)]=0, \\
& e^{-i \beta}[(\kappa+1) g+(\kappa-1) \mathbf{S}(g)]-e^{i \beta}[(\kappa+1) \bar{g}-(\kappa-1) \mathbf{S}(\bar{g})]=0 .
\end{aligned}
$$


By introducing the following notations

$$
g=\mathrm{M}+i \Lambda, \quad \mathrm{M}^{\prime}=\mu,
$$

we present (36) in the form

$$
\begin{aligned}
& \sin (\alpha) \mathrm{M}^{\prime}+i \cos (\alpha) \mathbf{S}\left(\mathrm{M}^{\prime}\right)=0, \\
& (\kappa+1) \cos (\beta) \Lambda-i(\kappa-1) \sin (\beta) \mathbf{S}(\Lambda)=f .
\end{aligned}
$$

Here the right hand side of the latter equation depends on the solution of the former equation. It has the form

$$
f=(\kappa+1) \sin (\beta) \mathrm{M}+i(\kappa-1) \cos (\beta) \mathbf{S}(\mathrm{M}) .
$$

The system of SIE (38) can be reduced to two Riemann BVPs on the real axis. The former SIE in (38) is homogeneous; it can be reduced to the following homogeneous Riemann BVP

$$
A^{+}=-e^{2 i \alpha} A^{-} .
$$

The latter equation in (38) is equivalent to the following non-homogeneous Riemann BVP

$$
B^{+}=e^{2 i(\beta-\varsigma)} B^{-}+\frac{e^{i \beta}}{\kappa+e^{2 i \beta}} f, \quad \varsigma=\arg \left(\kappa+e^{2 i \beta}\right) .
$$

The indexes of these BVPs are determined as follows

$$
\begin{gathered}
2 \mathrm{~K}=\operatorname{Index}\left(e^{2 i \alpha}\right)=\operatorname{Index}\left(e^{-4 i \theta}\right)=-\left.\frac{2}{\pi}[\theta]\right|_{-\infty} ^{\infty}, \\
2 \mathrm{~N}=\operatorname{Index}\left(e^{2 i \beta}\right)=\left.\frac{1}{\pi}[\beta]\right|_{-\infty} ^{\infty} .
\end{gathered}
$$

In (43) it has been taken into account that $\operatorname{Index}\left(e^{i \zeta}\right)=0$ as $\operatorname{Re}\left(\kappa+e^{2 i \beta}\right)>0$.

Further on we employ the general solution from Gakhov [7] to seek solutions of (40)-(41) vanishing at infinity. These solutions obviously depend on the indexes (42)-(43) respectively. The solution for the function $\mathrm{M}$ is found in a similar way as (18). It has the form

$$
\mathrm{M}^{\prime}(x)=2 \cos \alpha(x) \frac{P_{2 \mathrm{~K}-1}(x)}{\left(x^{2}+1\right)^{\mathrm{K}}} e^{-\mathrm{S}\left[\mathrm{K} \ln \left(\frac{t-i}{t+i}\right)+i a(t)\right]} .
$$

Here the exponential function is real-valued, thus the coefficients of the polynomial $P_{2 \mathrm{~K}-1}$ should be real as well to provide real-valuedness of the sought function. Therefore for positively defined index (42) the solution depends on $2 \mathrm{~K}$ arbitrary real constants.

Let us solve the second SIE in (38) assuming provisionally that $2 \mathrm{~N} \geq 0$ and the function $f$ specified by (39) is known. This solution (vanishing at infinity) is found from the general solution provided by Gakhov [7] in the form

$$
\Lambda(x)=\cos (\beta(x)-\varsigma(x)) f(x)+i \sin (\beta(x)-\varsigma(x))\left[Z(x) \mathbf{S}\left(\frac{f(t)}{Z(t)}\right)-\frac{\tilde{P}_{2 \mathrm{~N}-1}(x)}{\left(x^{2}+1\right)^{\mathrm{N}}}\right] .
$$

Here for briefness the following notation was introduced

$$
Z(x)=\exp \left[-\mathbf{S}\left(\mathrm{N} \ln \frac{t-i}{t+i}-i \beta+i \varsigma\right)\right]
$$


It should be noted that this function is real-valued because the singular integral is real-valued. Then it becomes evident that the first term in the square brackets is purely imaginary, therefore, the second term should be purely imaginary as well to provide real-valuedness of the sought function $\Lambda$. Hence all the coefficients of the polynomial $\tilde{P}_{2 \mathrm{~N}-1}(x)$ are purely imaginary.

The general number of arbitrary real constants entering the solution is $2 \mathrm{~K}+2 \mathrm{~N}+1$, where we added an extra constant generated by integration of (44) in order to find the right hand side of the non-homogeneous equation in the system. This number is the maximum possible number of arbitrary constants provided that both indexes are positive. If one of them is negative, say $2 \mathrm{~N}<0$, then solutions exist if $(-2 \mathrm{~N}-1)$ integral conditions are imposed on the right hand side of the non-homogeneous equation of the system (see detail in Gakhov [7]). They can be always satisfied provided that $2 \mathrm{~K}>-2 \mathrm{~N}-1$. Therefore for solvability of the system of SIE (38) it is necessary and sufficient that the cumulative index $2 \mathrm{~K}+2 \mathrm{~N}+1$ is positive. Even more the number of solutions is an odd number because both indices determined by formulas (42) and (43) are even numbers, which is evident from the fact that the stress and displacement orientations attaint their initial values after the complete traverse of the contour.

It should be noted that the number of arbitrary constants affecting the stresses can be reduced to $2 \mathrm{~K}+2 \mathrm{~N}$, bearing in mind that three constants can be eliminated as translation and rotation of the rigid body do not affect stresses. As soon as the displacements at infinity were zero one needs to satisfy the only additional condition on rotation, which would fix one of the coefficients of the polynomial $\tilde{P}_{2 \mathrm{~N}-1}(x)$ from the condition $\operatorname{Im}(\Phi(0))=0$.

\section{CONCLUSIONS}

In this paper we investigated solvability of three non-classical boundary value problems for elastic half-plane formulated in terms of the given orientations of the displacements and stresses on the $x$-axis. This study complements the results obtained earlier (Section 3) and presents two novel solutions in the closed form (Sections 4 and 5). The latter are obtained by successive solution of the equations forming the system of SIE.

\section{ACKNOWLEDGEMENTS}

The author is thankful to the Russian Foundation for Basic Research that supported this work by the Grant 16-01-00260.

\section{REFERENCES}

[1] Galybin, A.N. \& Mukhamediev, Sh.A., Plane elastic boundary value problem posed on orientation of principal stresses. Journal of the Mechanics and Physics of Solids, 47(11), pp. 2381-2409, 1999. https://doi.org/10.1016/s0022-5096(99)00032-0

[2] Galybin, A.N. \& Mukhamediev, Sh.A., Integral equations for elastic problems posed in principal directions: application for adjacent domains. WIT Transactions on Modelling and Simulation. 42, WIT Press, Southampton, UK, pp. 51-60, 2006.

[3] Galybin, A.N., Boundary value problems posed in terms of stress orientations for plane crack systems. Proceedings of 12th International Conference on Fracture. Ottawa, on CD-ROM, paper T02.007 (fin 00560), 2009.

[4] Galybin, A.N., Boundary value problems of plane elasticity involving orientations of displacements and tractions. Journal of Elasticity, 102, pp. 15-30, 2011.

https://doi.org/10.1007/s10659-010-9259-4 
[5] Galybin, A.N., Boundary integral equations for plane elastic problems posed in terms of stress orientations. Proceeding of the 8th UK Conference on Boundary Integral Methods (Ed. D. Lesnic), University of Leeds, UK, 2011.

[6] Galybin, A.N., On solvability of the BVP formulated in terms of displacement orientations on the interface between dissimilar elastic materials. International Journal of Computational Methods and Experimental Measurements, 5(3), pp. 369-376, 2017. https://doi.org/10.2495/cmem-v5-n3-369-376

[7] Gakhov, F.D., Boundary value problems. Moscow, Nauka (3rd Russian edition, see also the translation of the first edition by Pergamon Press, 1966), 1977.

[8] Muskhelishvili, N.I., Some Basic Problems of the Mathematical Theory of Elasticity. P. Noordhoff, Groningen, The Netherlands, 1963. 